\title{
QUALITY OF LIFE IN PATIENTS WITH SPINE TUMORS
}

\author{
Olga Y. Shchelkova ${ }^{1}$, Aslan K. Valiev ${ }^{2}$, Ekaterina B. Usmanova ${ }^{3}$, \& Evgeny A. Sushentsov ${ }^{2}$ \\ ${ }^{1}$ St. Petersburg State University, Saint Petersburg, (Russia) \\ ${ }^{2}$ N.N. Blokhin Russian Cancer Research Center, Moscow (Russia) \\ ${ }^{3}$ International Preschool, Moscow (Russia)
}

\begin{abstract}
Introduction: Quality of life (QoL) in chronic somatic diseases is seen as integral characteristic of physical, psychological, emotional and social functioning of patient. QoL in oncological patients is studied intensively. Patients with spine tumors previously had poor prognosis. Modern surgical treatment in patients with spine tumors allows to increase survival rate. Consequently the issue of QoL in patients with spine tumors becomes especially actual.

The aim of the study was to reveal basic parameters of QoL in patients with spine tumors before and after surgical treatment.

Results: Study participants were 25 patients with spine tumors. The diagnosis was spine metastases from different primary sources. The methods were: VAS and Watkins scales, «SF- 36 Health Status Survey», Quality of Life Questionnaire-Core 30 of European Organization for Research and Treatment Cancer, Spine Oncology Study Group Outcomes Questionnaire. All patients had pain syndrome before treatment. Pain assessment using VAS and Watkins scales before and after treatment revealed pain reduction as a result of surgery. Patients with spine tumors assess their QoL in general after surgery significantly higher than before treatment. As well patients report increasing QoL after surgery on the parameters of neurological status and pain intensity which corresponds to VAS and Watkins scales assessment. Besides the results revealed improving of QoL in patients with spine tumors after treatment on the parameter of emotional functioning. The diagnosis of patients with spine metastases refers to last stages of the disease. So treatment in these patients in most cases initially aimed at palliative care helps not only reduce pain intensity but also increase their QoL and in this way allow patients have emotionally rich life.

Conclusion: Nowadays patients with spine tumors undergo effective surgical treatment which increases their survival rate and significantly improve their QoL.

The further research is needed to study psychological factors of QoL in patients with spine tumors in order to make relevant programs of functional, social and psychological rehabilitation for these patients.
\end{abstract}

Keywords: Quality of life, spine metastases, pain syndrome.

\section{Introduction}

The relevance of treatment in patients with spine metastases increased simultaneously with increasing in number of these patients in clinical practice. The issue of choosing the optimal method of treatment for patients with spine metastases is one of the most difficult and opened at the moment. The main criteria for choosing the method of treatment are patients' survival rate and quality of life (QoL). For patients with poor prognosis the only criterion for choosing treatment is QoL.

QoL in chronic somatic diseases is seen as integral characteristic of physical, psychological, emotional and social functioning of patient. QoL concept pays attention to emotional experience of disease in patients, particularly to personal sense of possibility to satisfy actual needs and possibility of full social functioning in spite of disease limitations.

\section{Patients characteristics}

Study participants since 2016 till 2018 (N=25) were inpatients of N.N. Blokhin Russian Cancer Research Center. The diagnosis was spine metastases from different primary sources.

All patients had surgical treatment. 


\section{Methods}

The methods were:

1. VAS and Watkins scales. VAS scale (Visual analog scale) was used for subjective assessment of pain by a patient. Watkins scale was used for an objective assessment of pain by a doctor.

2. SF- 36 Health Status Survey (Ware et al., 1993; Wasserman et al., 2011)

3. Quality of Life Questionnaire - Core 30 of European Organization for Research and Treatment Cancer (Aaronson et al., 1993; Fayers et al., 1995);

4. Spine Oncology Study Group Outcomes Questionnaire (SOSG-OQ) (Janssen SJ. et al., 2017).

\section{Results}

Study revealed that all patients with spine tumors had pain syndrome before treatment. Pain assessment using VAS and Watkins scales before and after treatment revealed pain reduction as a result of surgery.

Besides the results revealed significant differences between QoL parameters in patients before and after surgical treatment. According data of SF-36 and SOSG-OQ patients with spine tumors assess their QoL in general after surgery significantly higher than before treatment, as well as patients assess their emotional functioning after surgical treatment significantly better than before surgery.

The results of studying general QoL and emotional functioning in patients with spine tumors after surgery compared with QoL before surgery are presented on Figure 1.

Figure 1. General QoL and emotional functioning in patients with spine tumors before and after surgery.

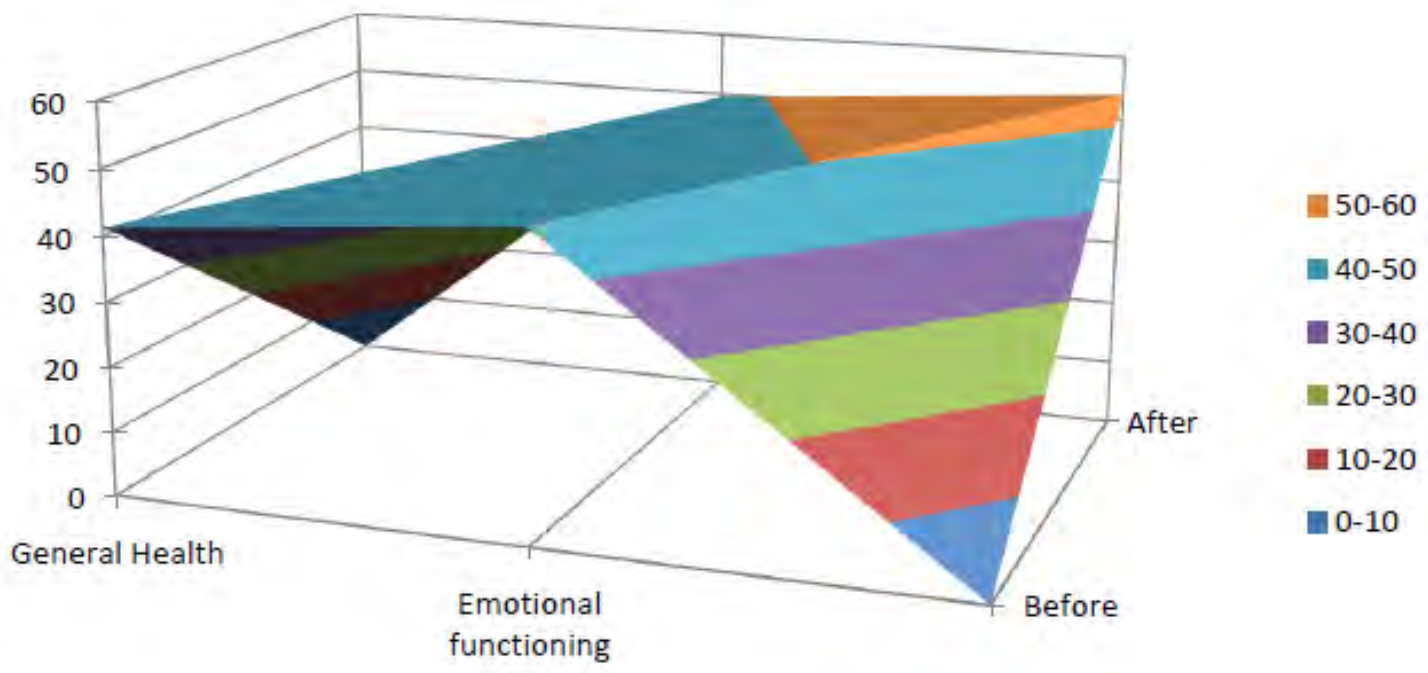

As well patients report increasing QoL after surgery on the parameters of neurological status and pain intensity according the results of SOSG-OQ and QLQ C-30.

The results of study neurological status and pain intensity in patients with spine tumors are presented on Figure 2. 
Figure 2. Neurological status and pain intensity in patients with spine tumors.

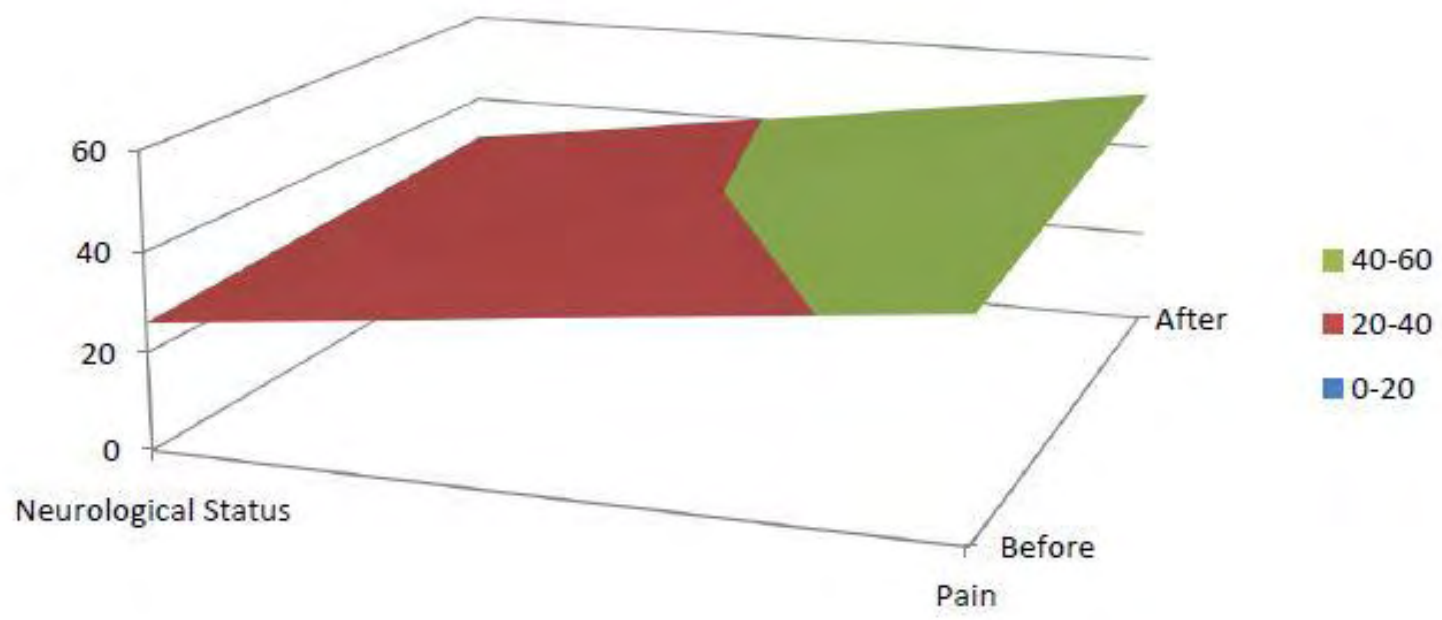

These results correspond to VAS and Watkins scales assessment.

In conclusion, patients with spine tumors assess their QoL on the basic parameters as general health, emotional functioning, neurological status and pain after surgery significantly higher than before treatment. Patients with spine tumors have an opportunity to undergo effective surgical treatment which increases their survival rate and significantly improve their QoL.

The further research is needed to study psychological factors of QoL in patients with spine tumors in order to make relevant programs of functional, social and psychological rehabilitation for these patients.

\section{Acknowledgements}

The research is supported with RFFI grant № 19-013-00154 A.

\section{References}

Aaronson, N. K., Ahmedzai, S., \& Bergman, B. (1993). The European Organisation for Research and Treatment of Cancer QLQ-C30: A quality of life instrument for use in international clinical trials in oncology. Journal of the National Cancer Institute, 85, 365-375.

Fayers, P., Aarson, N., Bjordal, K., \& Sullivan, M. (1995). QLQ C-30 Scoring Manual. Brussels: EORTC Study Group on Quality of Life.

Janssen SJ., Teunis T., van Dijk E. Validation of the Spine Oncology Study Group-Outcomes Questionnaire to assess quality of life in patients with metastatic spine disease // Spine J. - 2017. V. 17(6): 768-776.

Ware, J. E., Snow, K. K., Kosinski, M., \& Gandek, B. (1993). SF-36 Health Survey Manual and Interpretation Guide. Boston, MA: New England Medical Center, The Health Institute.

Wasserman, L. I., Trifonova, E. A., \& Shelkova A. Yu. (2011). Psychological diagnostics and intervention in somatic clinic. Saint Petersburg: Rech. 